
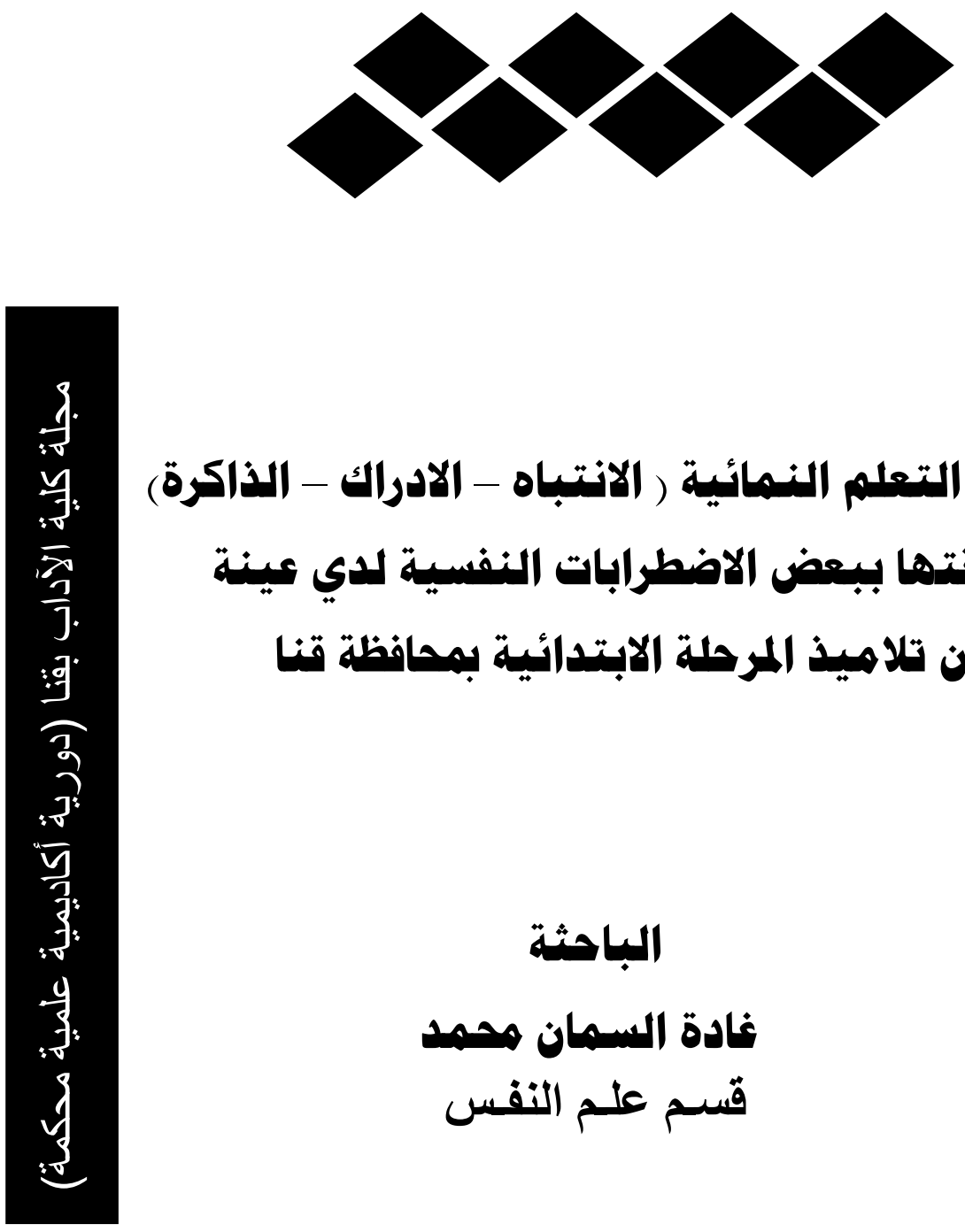

صعوبات التعلم النسمائية ( الانتباه - الادراك - الذاكرة)

وعلاقتها ببعض الاضطرابات النفسية لدي عينة

هن تلاهيذ المرحلة الابتدائية بممافظة قنا

\title{
الباحثة
}

غادة السمان همهمد

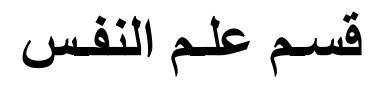


ان مفهوم صعوبات التعلم مصطلح عام يصف مجموعة متنوعة من مشكلات

التعلم وتلك الصعوبات تؤدي إلي اضطراب في التعلم وفي استخدام مهارات القراعة ، الكتابة، الفهم، النطق، والاستدلال وإجراء العمليات الحسابية، وقد نجد طفلاً يعاني من صعوبة في القراعة والكتابة، وطفلاً اخر قد يكون لايه صعوبة في الرياضيات، وطفلاً ثالثا قد يعاني من صعوبة في كل الجوانب السابقة، أو في فهم ماذا يقول الاخرون.

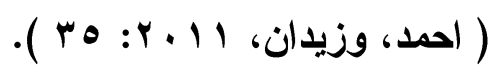

وتثير كثير من الدراسات والبحوث التي اجريت في مجال صعوبات التعلم إلي

وجود علاقات وثيقة بين صعوبات التعلم النمائية وصعوبات التعلم الأكاديمية وصعوبات السلوك الاجتماعي والانفعالي، كما تشير الملاحظات الميدانية المباشرة إلي تدعيم وجود هذه العلاقات، وقد لوحظ خلال العمل في الإشراف علي التربية الميدانية ببعض الدول العربية نوعاً من التجاهل غير المبرر في الأنظمة التعليمية العربية لمجال صعوبات التعلم النمائية ولكن انصب الاهنمام علي صعوبات التعلم الأكاديمية دون صعوبات التعلم النمائية وصعوبات السلوك الاجتماعي، وحيث نري أن صعوبات التعلم النمائية هي منشأ صعوبات

التعلم الأكاديمية ( Ernest Siegel , Ruth F. Gold, 1982, 122 )

Introduction:

The concept of learning difficulties is a general term describing a variety of learning problems and these difficulties lead to a learning disorder and to the use of reading skills, writing, comprehension, pronunciation, reasoning and calculations, and we may find a child with difficulty reading and writing, and another child may be He has difficulty in mathematics, and a third child may have difficulty in all previous aspects, or in understanding what others say.

Many of the studies and research conducted in the area of learning disabilities indicate close relationships between developmental learning disabilities, academic learning disabilities and difficulties of social and emotional behavior, and direct field observations indicate that the existence of such relationships is being strengthened, and it has been observed during work in Supervision of field education in some Arab countries is a kind of unjustified disregard in the Arab educational systems for the field of developmental learning difficulties, but attention focused on academic learning difficulties without the difficulties of developmental learning and difficulties of social behavior, and where we see that learning difficulties Developmental is the origin of academic learning disabilities. 
أولاً: صعوبات التعمّ

حيث يعتبر "صموئيل كيرك" أول من أثار إلى مصطلح صعوبات التعلم وصرح بأنه إعاقة خاصة أو قصور في واحدة أو أكثر من عمليات النطق أو اللغة والإدراك والقراعة والكتابة والعمليات الحسابية وهى ناتجة عن احتمال وجود خلل بسيط في الاماغ أو اضطر اب انفعالي أو سلوكي ولكنها ليست ناتجة عن التخلف العقلي أو الحرمان الحسى وهى وهي ( السمع والبصر) ، وقد تعزى إلى مسببات ثقافية أو طرق التدريس.( تيسير كوافحة

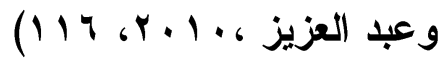
التعريف الفيدر المي لصعوبات التعلم : أصدرت الحكومة الفيدر الية الأمريكية تعريف صعوبات التعلم من خلال تحديده في

ثلاث نقاط كما يلي: من الناحية العامة : يعني مصطلح صعوبة التعلم النوعية أو المحدة وجود اضطر اب في واحدة أو أكثر من تلاك العمليات السيكولوجية الأساسية المستخدمة في فهم أو استخدام اللغة سواء المكتوبة أو المنطوقة ، وهو الاضطر اب الأي يظهر في شكل قصور في قرة الطفل علي الاستماع أو التفكير، أو التحدث أو الكتابة أو الهجاء، أو إجراء العمليات

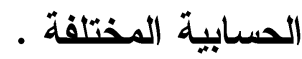

من ناحية الاضطرابات المتضمنة': يتضمن هذا المصطلح حالات مثل: صعوبات الإدراك وإصابات الاماغ واختلال الأداء الوظليفي وعسر القراعة ، والحبسة الكلامية

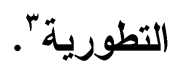
الاضطرابات غير المتضمنة: : لا يتضمن مثل هذا المصطلح مشكلات التعلم التي تعتبر في أسساسها نتيجة لإعاقة بصرية أو سمعية ، أو حركية ، أو تخلف عقلي، أو اضطراب انفعالي أو قصور بيئي، أو ثقافي ، أو اقتصادي بعاني الطقل منه.

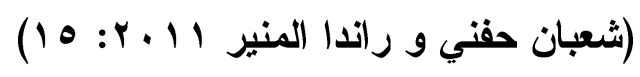

تعرف نتاليا و تناتينا ( Natali M \& Tatiana, 2012, 49 ) صعوبات التعلم:

هي تأخير جزئي في نمو الوظائف العقلية العليا وتستطيع المناهج النفسية العصبية أن تقيس حالة هذه الوظائف، وايضاً توضح روئة علم النفس العصبي ان هؤلاء الأطفال

\footnotetext{
${ }^{1}$ Disorders Involved

${ }^{2}$ Minimal brain dysfunction

${ }^{3}$ Developmental Aphasia

${ }^{4}$ Disorder not involved
} 
لايهم مشكلات في القراعة، الكتابة وحل الحساب، وأيضا في الكلام الثفهي وفهمه وهذا

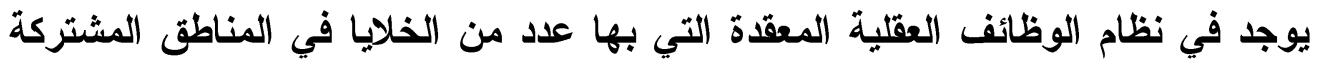

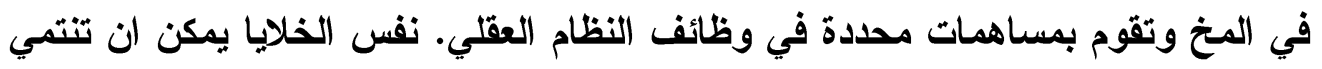

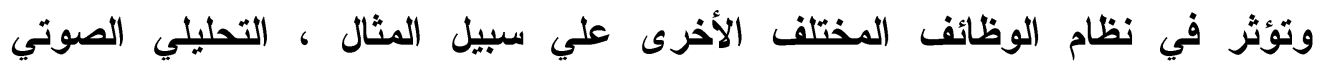

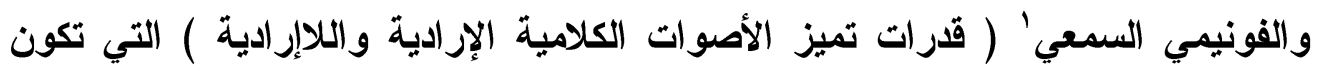

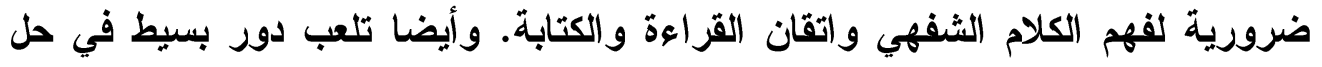

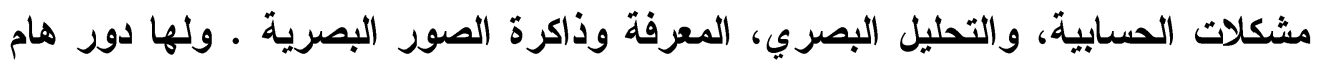
في القراءة.

يعرف هاميل و لا رسين ( Hammill \& Larsen, 1981, 82 ) صعويات التعلم هي مجموعة غير متجانسة من الاضطرابات التي ظهرت بواسطة صعات صعوبات محدده في

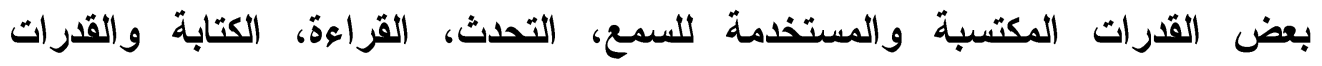
الحسابية. هذه الاضطر ابات تعتبر جوهرية عند الاطفال ويفترض انها تظهر بسبب اختلال

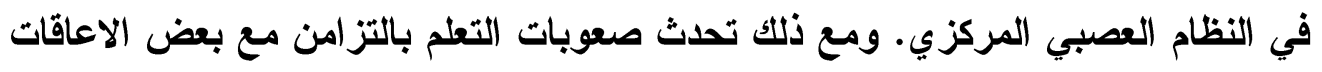

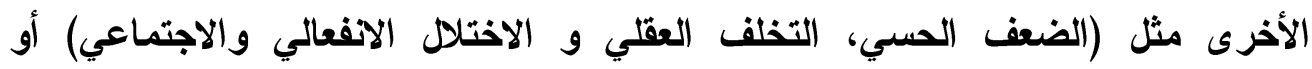

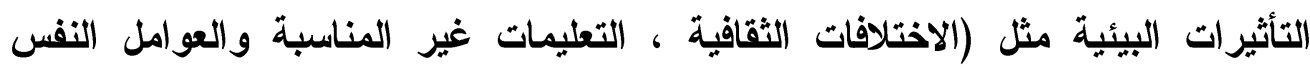
جينية) و انها ليست نتيجة مباشرة لهذه الظروف أو التأثيرات.

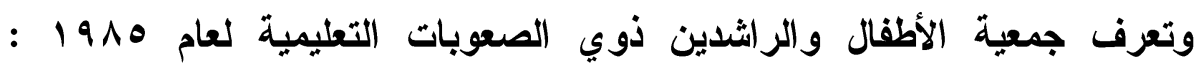

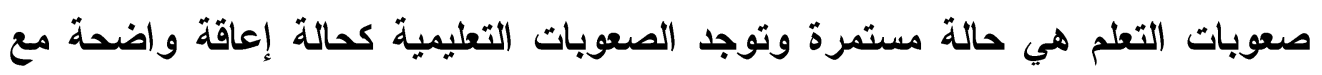

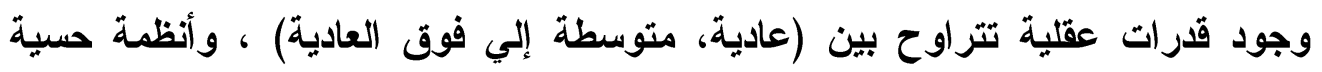

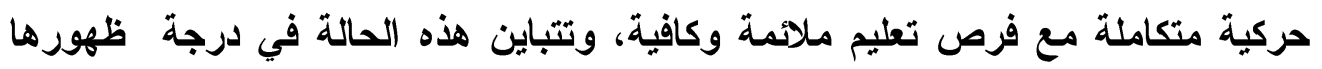
وفي درجة شدتها ويمكن لهذه الحالة أن تؤثر مدي الحياة علي تقدير وتهائ الفرد لذاته،

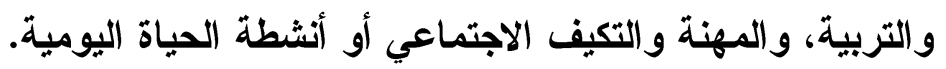

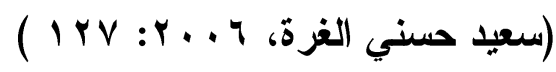

الفرق بين صعوبات التعلم وبطء التعلم والتأخر الار اسي:

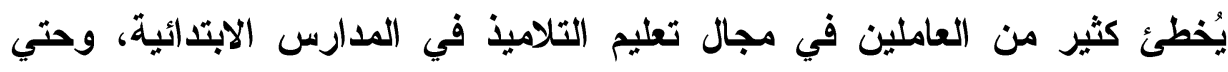
بعض المسئولين الإداريين العاملين في حقل التربية الخاصة في التمييز بين صعوبات

${ }^{1}$ Phonetic Audio Analysis 
التعلم، وبطء التعلم والتأخر الاراسي اذ يستخدمون مصطلح من هذه المصطلحات وكأنه

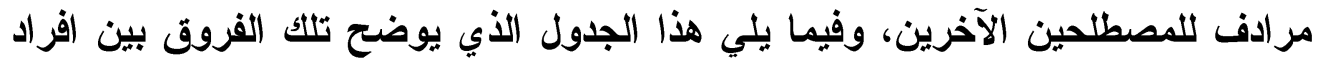
الفئات الثلاثة المشار اليها:

جدول رقم (1) الفرق بين التلاميذ الذين يعانون من صعوبات التعلم، وبطيئي التعلم والمتأخرين دراسياً

\begin{tabular}{|c|c|c|c|}
\hline التأخر الدرسي & بطء التعلم & صعوبات التعلم & مظاهر \\
\hline منخفض في جميع المو اد & مثنفض في الاستيعاب، مع عدم القدرة & منذفض في المواد التي تحتوي & التحصبل الدراسي \\
\hline ضعف الدافعية للتعلم & انخفاض نسبة الأك & اضطراب في العمليات الأهنية & سبب التدني \\
\hline نسبة (الذكاء عادية أو مرتفعة & 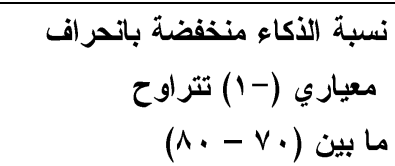 & نسبة الأكاء عادية أو مرتفعة & (القدرة (العقلية \\
\hline مرنبط بسلوكيات غير مرغوبة أو & مشاكل نسبية بسبب عوامل بيئية & اعتيادي، وقد يصحبه نشاط زائد & المظاهر السلوكية \\
\hline تقريم خدمات الارشاد التربوي من مختص في تقديم مثل هذه & 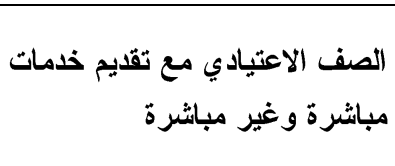 & برنامج خاص بصعوبات التعلم & 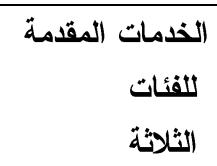 \\
\hline
\end{tabular}

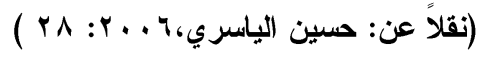

ثانياً صعوبات التعلم النمائية 1

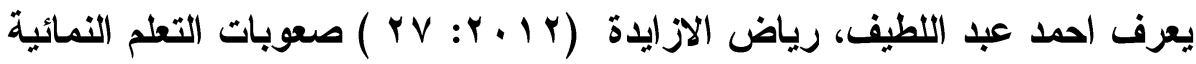

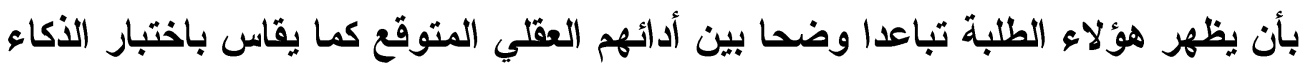

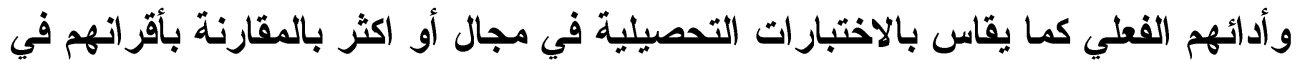
نفس العمر الزمني والمستوي العقلي والصف الدراسي.

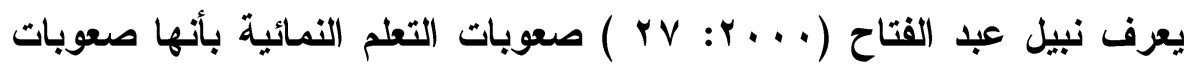

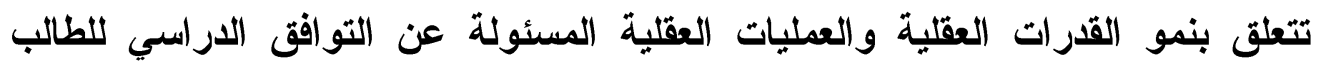
وتو افقه الثخصي والاجتماعي والمهني وتثنمل:- صعوبات الاتتباه والإدرالك والتفكير

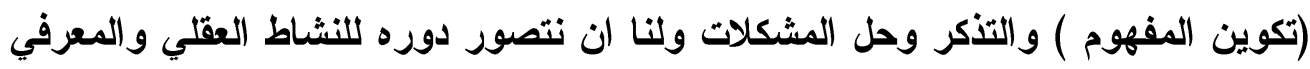
تعد مسؤولة عن اكتساب الخبرة التربوية عن طريق الوسائل التعليمية وغير التعليمية

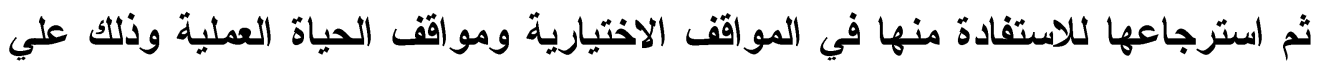
النحو التالي: 


\section{الادر اك}

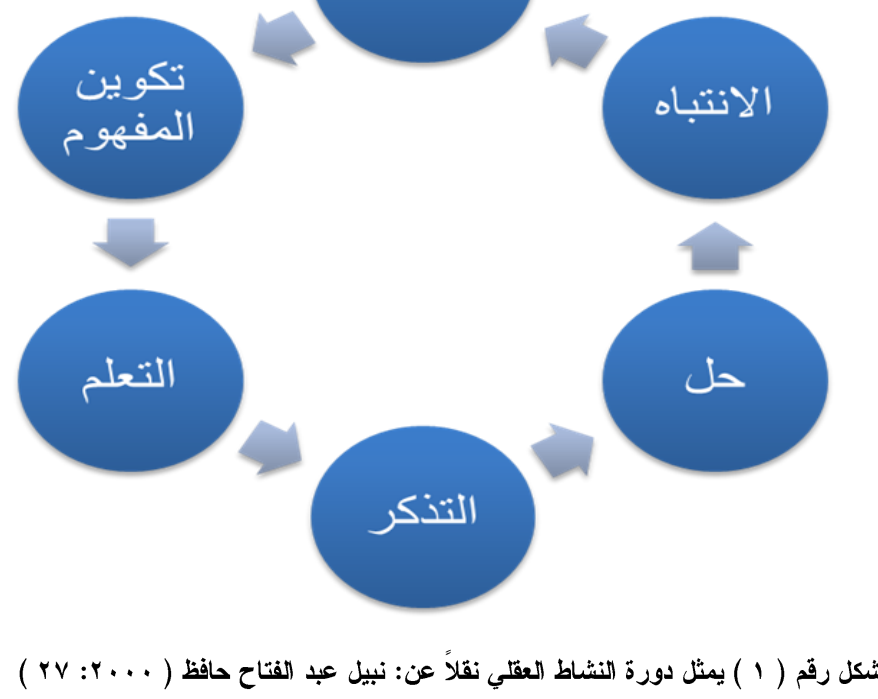

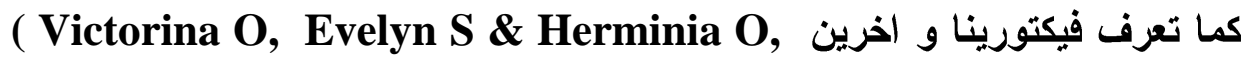
2004:117, 118 )

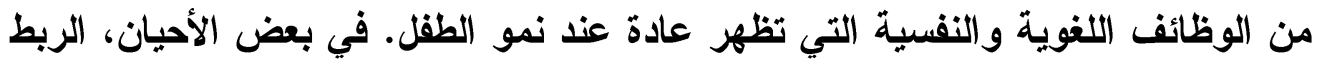
بين الصعوبات التعلم النمائية والصعوبات الاكاديمية هو يصف العبه العجز في المهارات

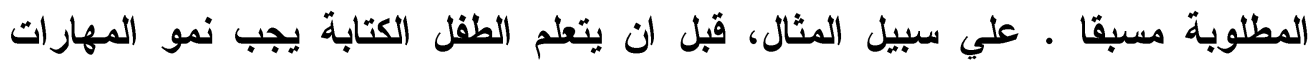
السابقة مثل التنسيق بين اليد والعين ، الأكرة ، وقدرات التسلسل ـ وفي تعلم القراعة

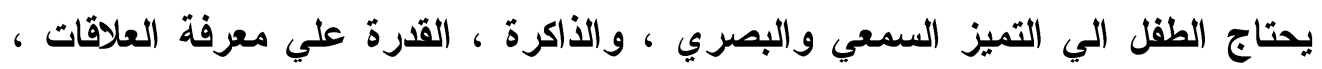

$$
\text { و والتعلم من تكرار الخبرات والقدرة علي تركيز الاتتباه. }
$$

1- اضطراب اللغة' : لا يستطيع الطقل التحدث مثل اشقائه الأكبر او من نفس العقر، او لا يستطيع الإجابة بثكل كاف او استخدام الجمل اللفظية.

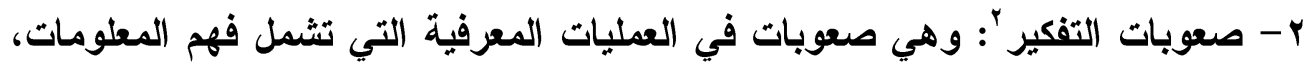
مهارة حل المشكلات و الربط بين الأفكار. وحل المشكلات تمثل واحدة من السلوك

\footnotetext{
${ }^{1}$ Language Disorder

${ }^{2}$ Difficulties of Thinking
} 
المعرفي في التسلسل الهرمي الأي يتطلب التحليل والتركيب للمعلومات ومساعدة الأفراد

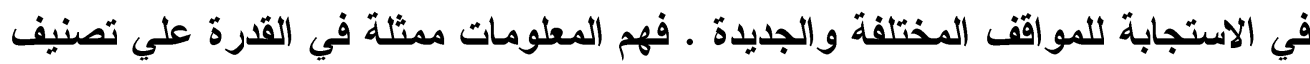

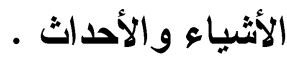

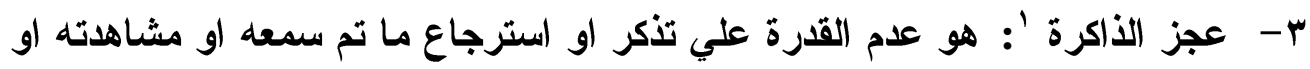

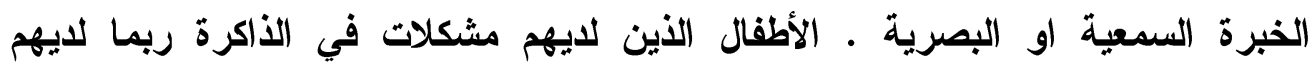
صعوبات في القراعة أو في استرجاع المظهر البصري ، والذاكرة السمعية التي ربما

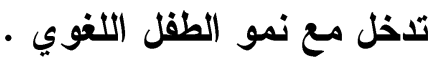
ـ - اضطراب الاتتباه ` ؛ انه نتيجة لتأخر نمو القدرة علي توظيف والحفاظ علي الاتتباه الانتقائي ، عندما الطقل يستجيب لكثير من المنبهات هو يعتبر متثتت.

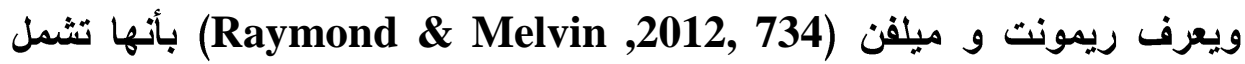
صعوبات في العمليات البصرية والسمعية، والوظائف الحركية، ودمج طرائق اللغة

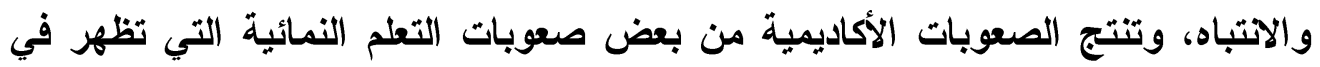

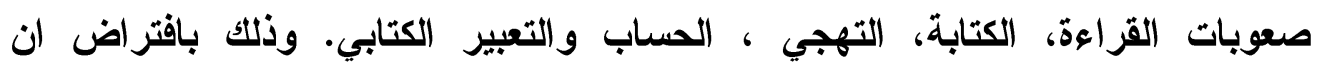
صعوبات التعلم النمائية منشأ صعوبات التعلم الأكاديمية.

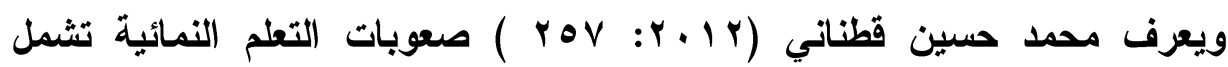

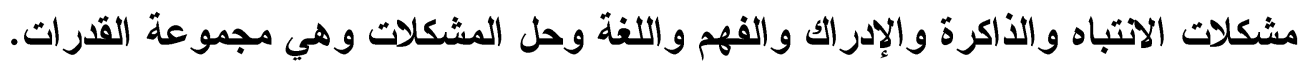

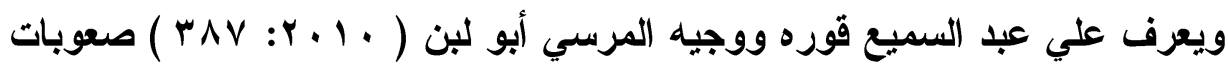
التعلم النمائية هي تنقسم الي قسمين: ا- صعوبات تعلم أولية ": وتثمل صعوبة الاتباه، صعوبة الإدرالك، صعوبة الذاكرة،

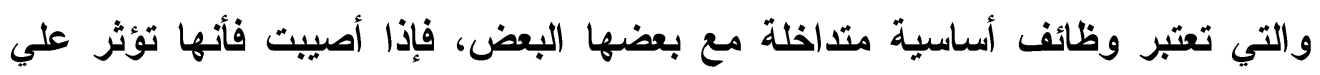
النوع الثاني من الصعوبات النمائية . r- صعوبات تعلم ثانوية؛ : وتثمل صعوبة في التفكير وصعوبة اللغة الثفوية، وصعوبة حل المشكلات.

\footnotetext{
${ }^{1}$ Memory Deficiency

2 Attention Disorder

${ }^{3}$ Initial Learning Disabilities

${ }^{4}$ Secondary Learning Disabilities
} 
وتثير كارلا (Carla ,2008, 22 ) صعوبات التعلم النمائية هي مرتبطة بالقدرات

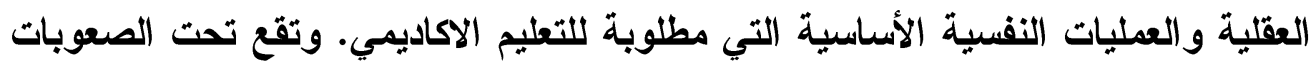

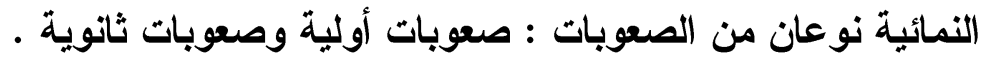

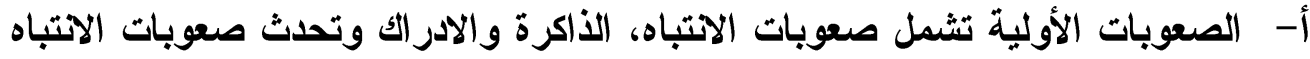
عندما الطلاب يتميزون بمشكلات في التركيز والمحافظة علي انتباههم مما يسبب التباه غير مستقر الأي ربما يحدث بسبب عدم الراحة الجسمية ، والمشكلات الانفعالية، والعو امل التحفيزية او التحديات ميع تنظيم الذات.

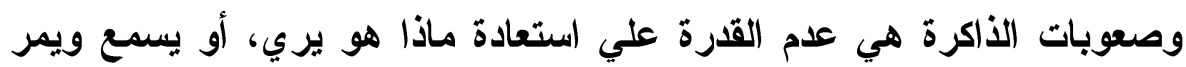
بخبرات. أما الصعوبات الادر اكية تثمل صعوبات في الاتساق البصري وات العلاقات التات المكانية.

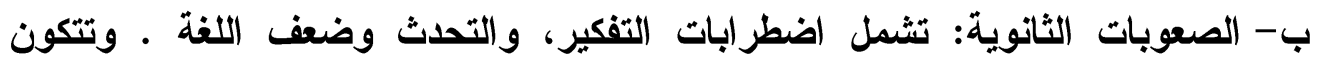
اضطرابات التفكير من مشكلات في العمليات العقلية التي تثمل العمليات الحسابية،

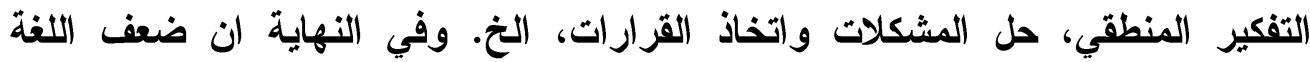
و التحدث مرتبط بالفهم وإنتاج اللغة الثفهية التي تكون عملية معقدة تثمل تدعيم الأفكار

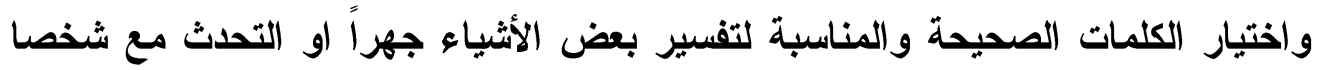
ما. ويعرض السيد عبد الحميد سليمان ( . . . . : 9 \& 1 ) ان صعوبات التعلم النمائية

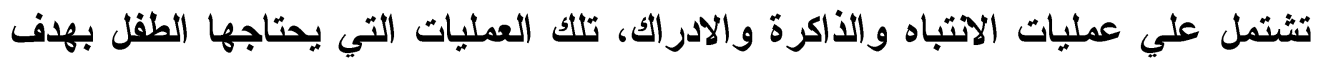

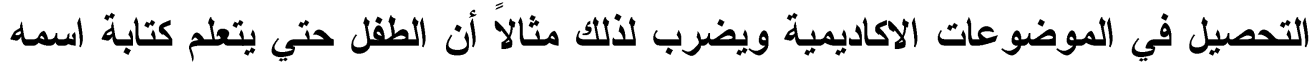

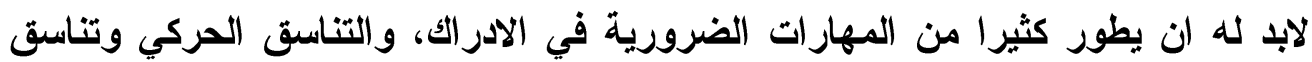

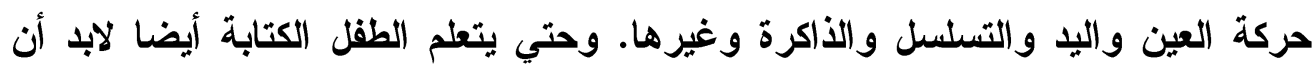

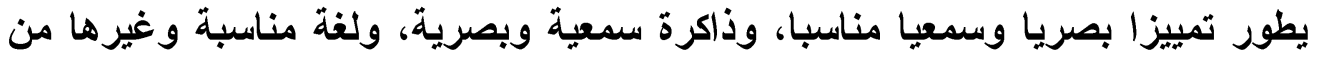

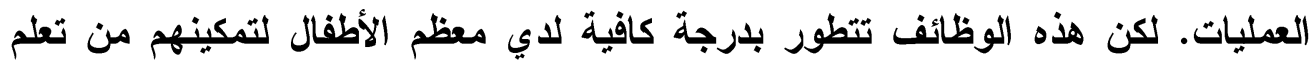

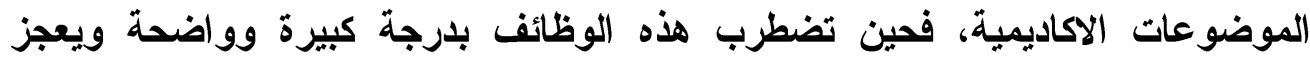
الطقل عن تعويضها من خلال وظائف اخري عندئذ تكون لايه صعوبة في تعلم الكتابة التهابة والتهجئة أو إجراء العمليات الحسابية.

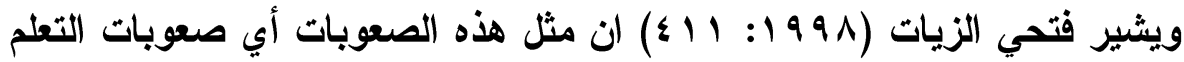
النمائية عادة ما تتدرج في أطار ثلاثي علي النحو التالي: 
1- الصعوبات المعرفية': ومظاهرها (حل المشكلات، الاتتباه، التمييز، وصعوبات

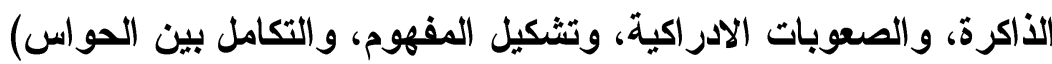

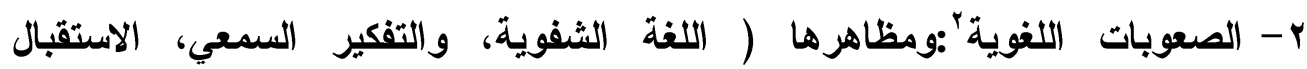
(السمعي) r- الصعوبات البصرية الحركية"ّ ومظاهرها (أداء مهارات حركية كبيرة تعكس التناست

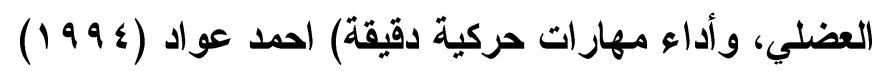

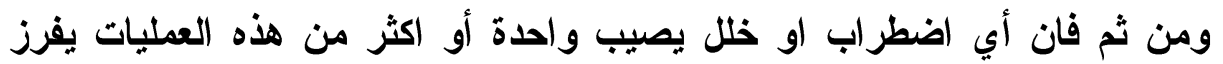

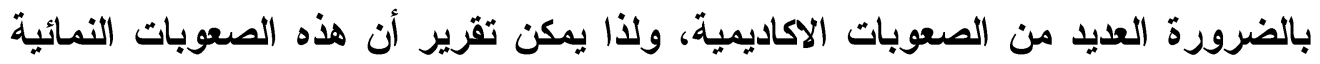

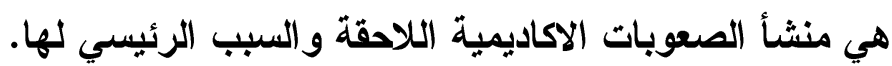
وتعرف الباحثة صعوبات التعلم النمائية نظرياً هي اضطر اب في الوظئي لهائف الوائف المعرفية

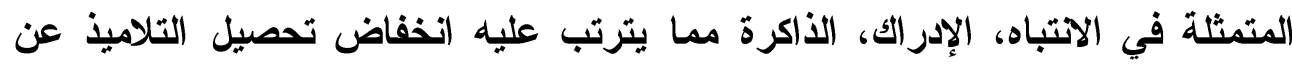

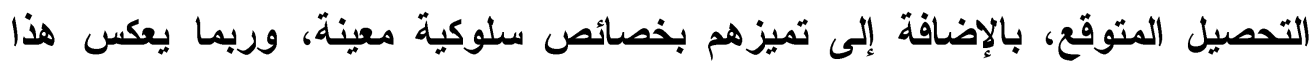

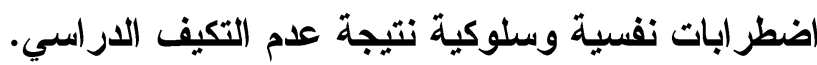

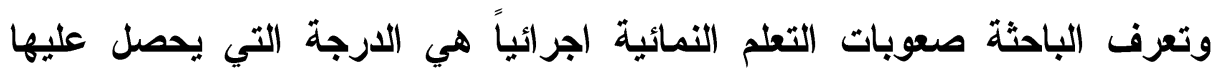

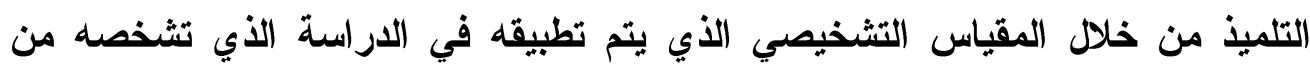
ذوي صعوبات العلم النمائية من اعداد الباحثة. العلاقة بين صعوبات التعلم النمائية وصعوبات اعلية التعلم الاكاديمية:

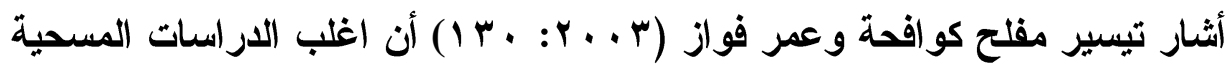
التي قامت حول العلاقة بين صعوبات التعلم النمائية وصعوبات التعلم الاكاديمية أثشارت

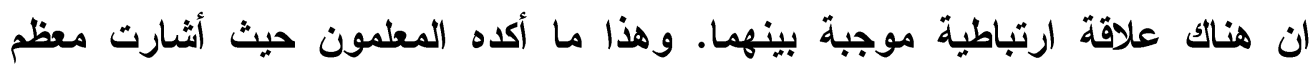
الاراسات أن المعلمون كانوا يؤكدون علي انه يجب الأخذ بعين الاعتبار وبشكل مؤكد أن أن أندان

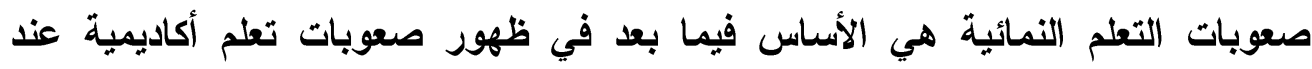

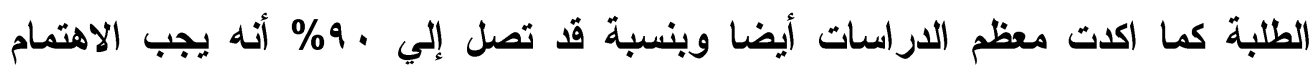
بالعمليات النفسية الأساسية عند الطلبة وكذلك بأساليب التدريس المستخدمة عند هؤلاء الهيه

${ }^{1}$ Cognitive Difficulties

2 Language Difficulties

3 Visual Optical Difficulties 
واكد فلتشر وفورمان (Fletcher \& Foorman 1994, 19) انه لكي نصل بفاعلية لعلاج ذوي صعوبات التعلم الأكاديمية إلي الحد الأقصى لها، يجب أن يكون التركيز علي الوقاية والتدخل المبكر بالنسبة لصعوبات التعلم النمائية.

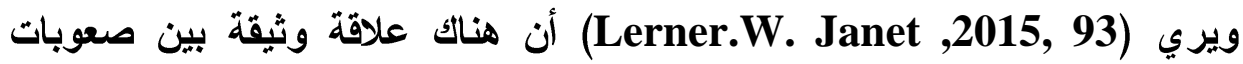

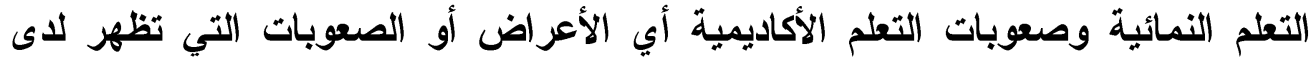

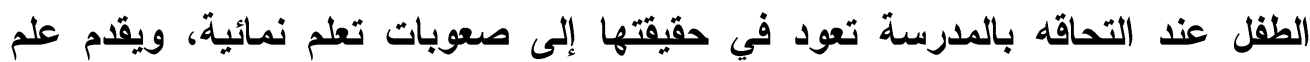

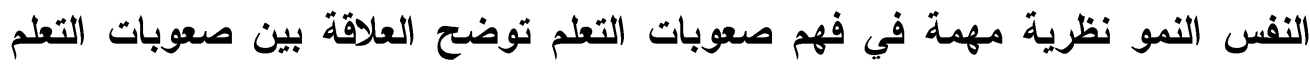

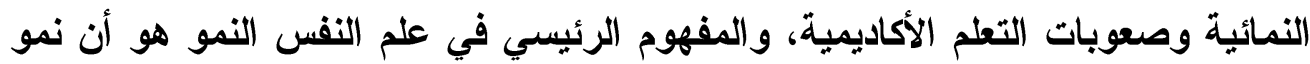

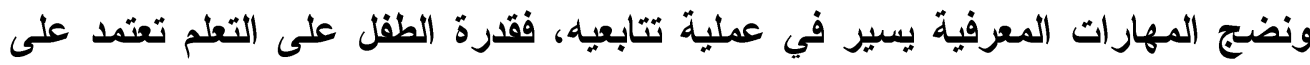

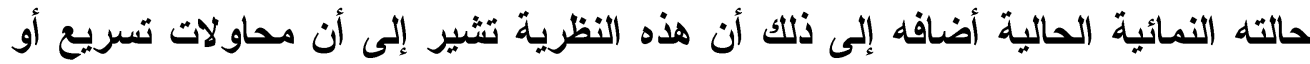

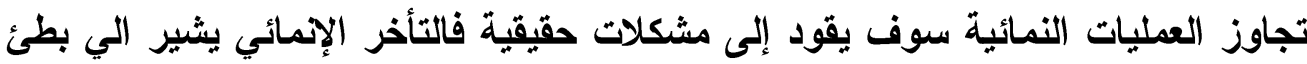

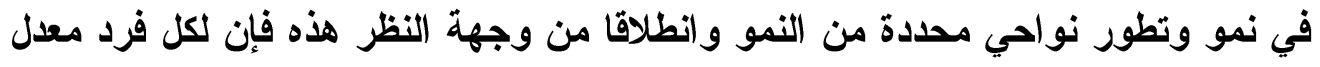

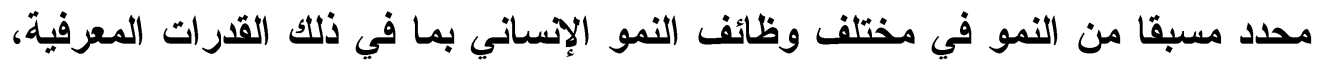

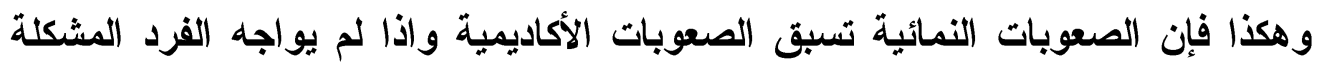
في النمو فلن تكن هنآك مشكلات أكاديمية.

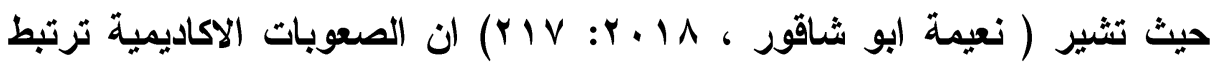
بالصعوبات النمائية فتعلم القراعة يتطلب القدرة علي فهم واستخدام المفردات اللغوية التواتية

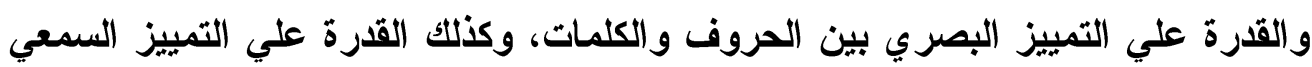

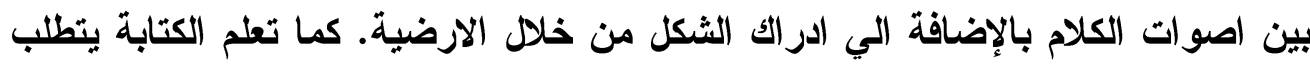

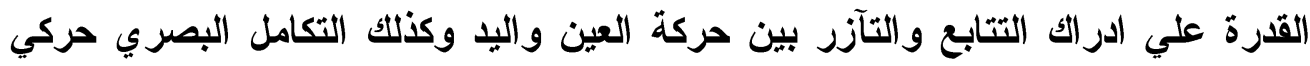
والذاكرة البصرية. إذن فالعلاقة بين صعوبات التعلم النمائية وصعوبات ولئ التعلم التاكئ الاكاديمية هي علاقة سبب ونتيجة حيث تثكل الأسس النمائية المحددات الرئيسية للتعلم الاكاديمي

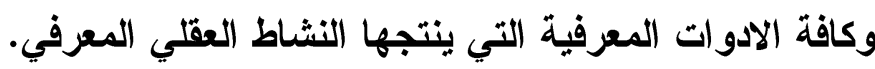

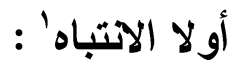
يشير اوميلتا (Umilta, 1998, 23:31) ان عملية الاتتباه هي احدي العمليات

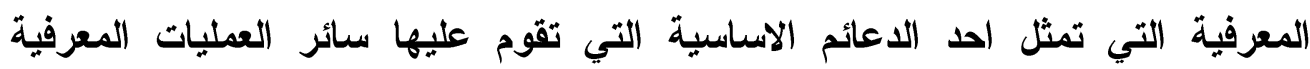

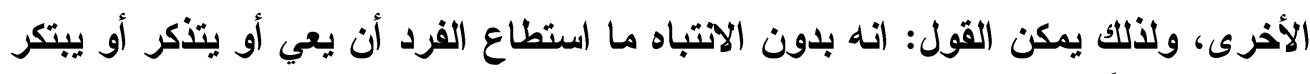
أو يتخيل شيئاً.

${ }^{1}$ Attention 


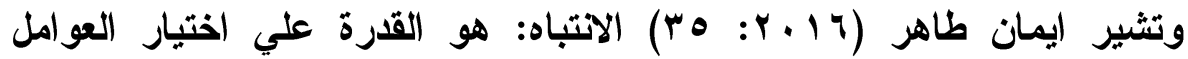
المناسبة، ووثيقة الصلة بالموضوع من بين مجموعة من المثيرات التي يصادفها الكائن

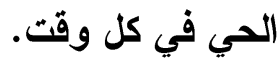

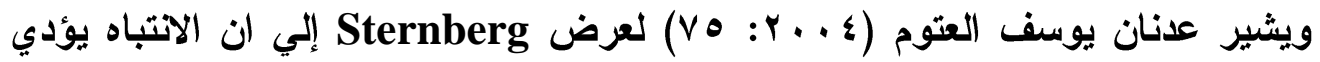
ثلاث وظائف وهي: 1- انتباه موجه' : وهو محاولة الفرد تعرف حدوث مثير حسي بالبيئة المحيطة به.

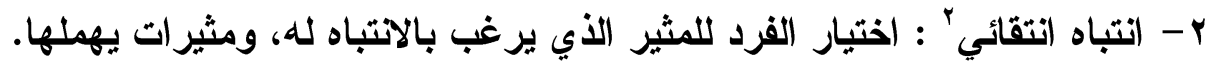

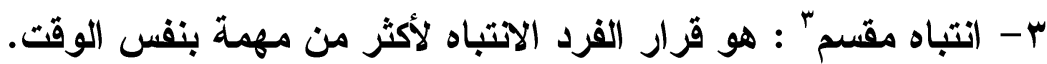

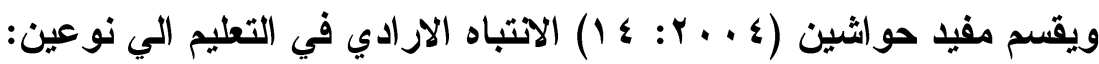

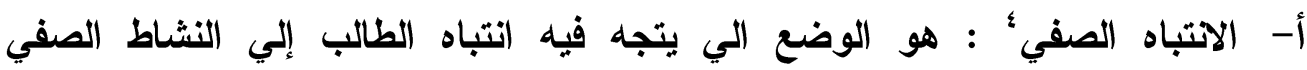
المستهدف وإهماله كافة المثيرات الأخرى غير المستهدفة كأصوات السيارات في الخارج أو الصور علي الجدران وغيرها. ب- الاتباه اثناء التعلم الذاتئ: وهو تركيز الانتباه علي موضوع المان التعلم إذا كان مطالعة

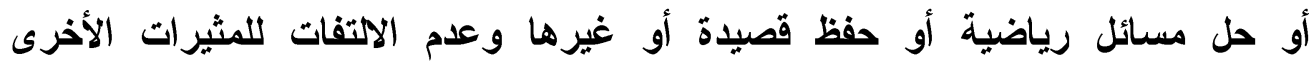
الموجودة في البيئة أو الاستغراق في أحلام اليقظة. وتعرف الباحثة الاتتباه نظرياً هو عملية عقلية اساسية تمثل قدرة الفئل الفرد علئي التركيز

$$
\text { صعوبات الاتتباه " الذهين. }
$$

يعرض ويندر (Wender, P.H, 1987) انها مجموعة من الخصائص السلبية المتعلقة بالانتباه لدي الأطفال مضطربي الاتتباه المصحوب بفرط النشاط وتتمثل في هذه من إن

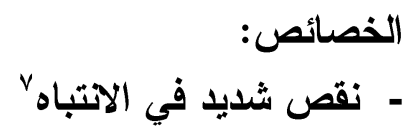
حيث يقل المدي الزمني للانتباه، فلا يستطيع الطقل تركيز انتباهه سوي لفتراه من الزمن، كما يصعب عليه التركيز الذهني اثناء العمل أو أداء المهام الداراسية.

\footnotetext{
${ }^{1}$ Attention Prompt

${ }^{2}$ Selective Attention

3 Attention Divider

${ }^{4}$ Descriptive Attention

${ }^{5}$ Attention During Self Learning

${ }^{6}$ Attention Disabilities

${ }^{7}$ Inattention
} 
حيث يعجز الطفل عن الاختيار الاتتقائي لمثير محدد في محيطه البصري، ولكن يتجه إلي

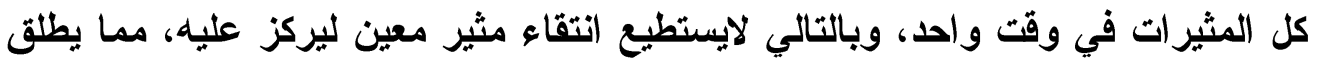

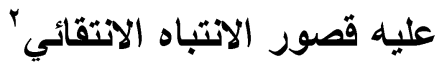
-

هو ميل الطقل للثبات والاستمرار في النشاط مدة طويلة بعد انتهاء الحاجة المنطقية

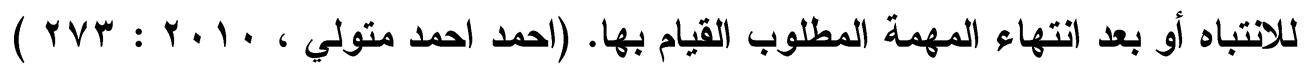

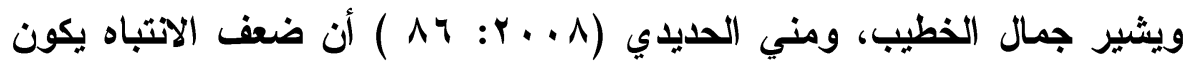

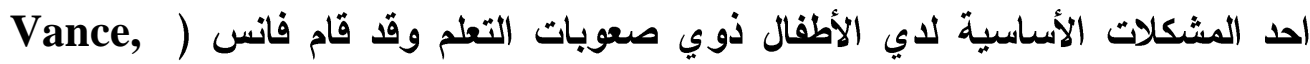

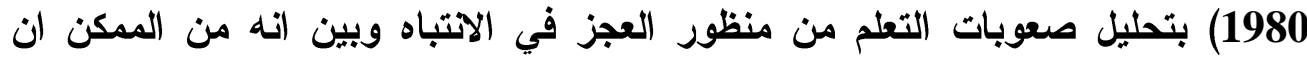

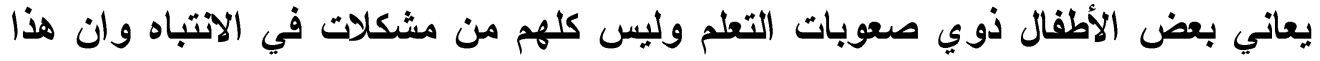

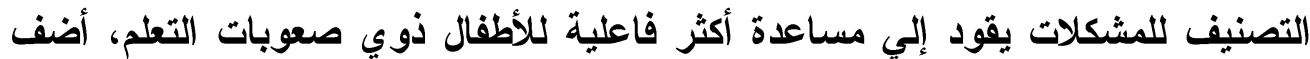

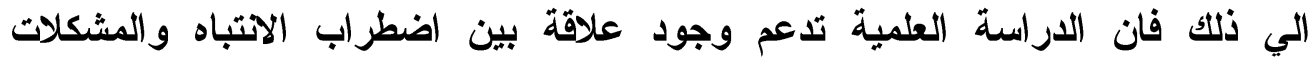

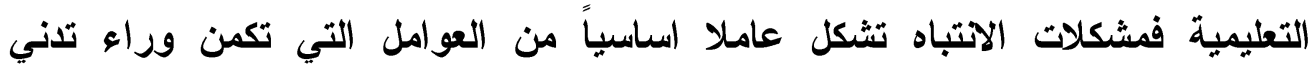

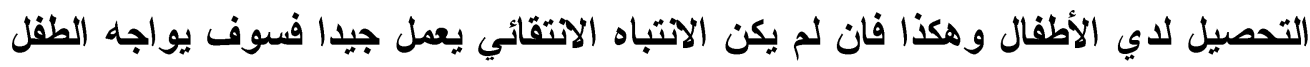

صعوبات في التعلم.

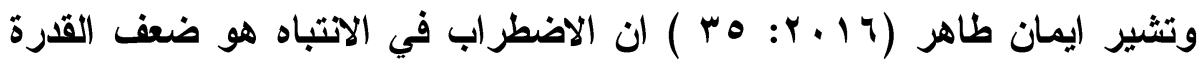

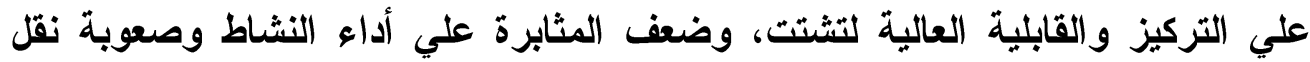

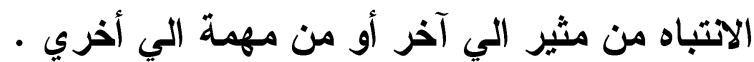

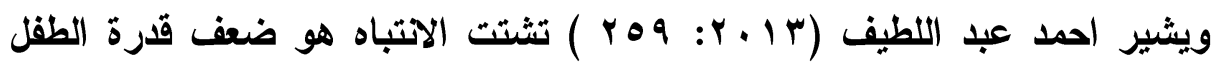

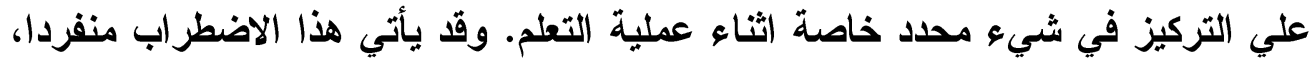
وقد يصحب بالنشاط الحركي الزائد والأدفاعية غير الموجهة.

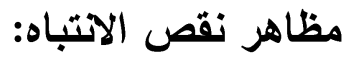

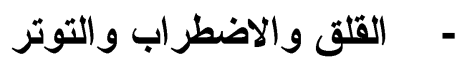

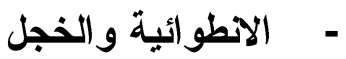

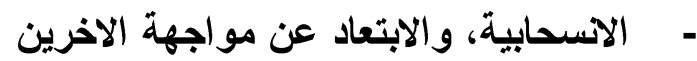
- قصر فترة الانتباه اثناء المهام المدرسية أو اثناء القيام بأي نشاط يحتاج التباه

\footnotetext{
${ }^{1}$ Attention Distractibility

${ }^{2}$ Selective Attention Deficits

${ }^{3}$ Attention Perseveration
} 
- ععوبة متابعة التوجيهات والارشادات الموجهة إليه. وكأنه لا يستمع إلي المتحدث.

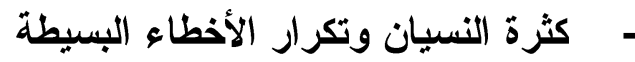
- - الظهور بمظهر من يحلم أحلام اليقظة. - - صعوبة التركيز لفترة طويلة ( شرود ذهني متكرر)

ثنانيا الذاكرة يثير عادل عبد الله (ع . . ب : 9 ) تعتبر الذاكرة بمثابة القدرة علي الاحتفاظ بما مر بالفرد من معلومات ومواقف وخبرات واحداث مختلفة متعددة وغيرها، ثم القيام

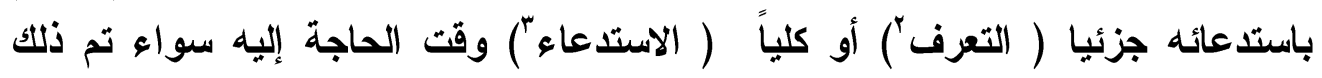
بصورة منسلسلة أو بصورة حرة.

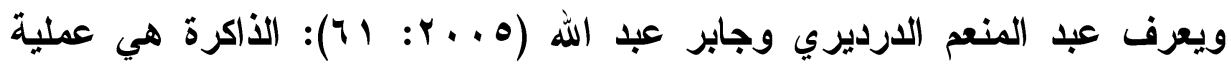
عقلية تفاعلية إنتاجية مستمرة تعمل علي تخزين واسترجاع المعلومات والخبرات.

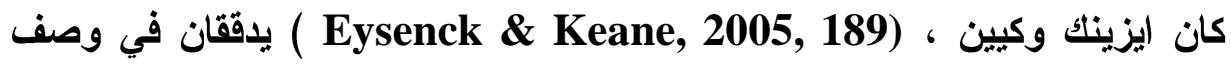

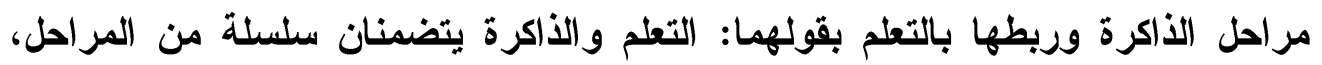

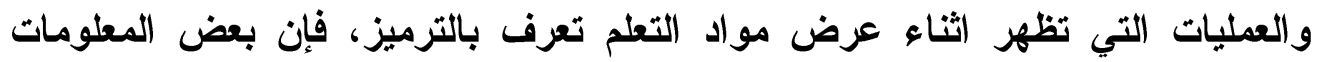

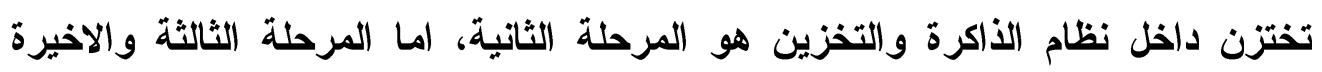
الاسترجاع وتتضمن استعادة المعلومات المخزنة في الأكرة.

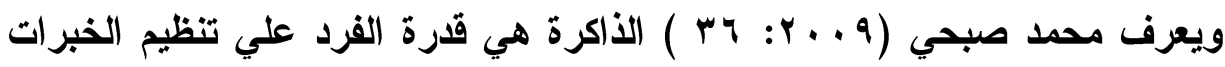

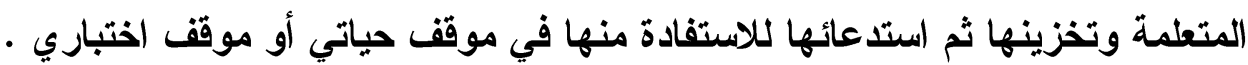

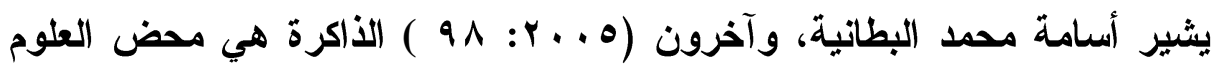

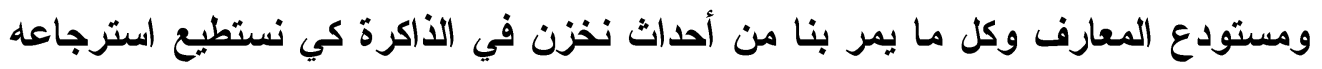

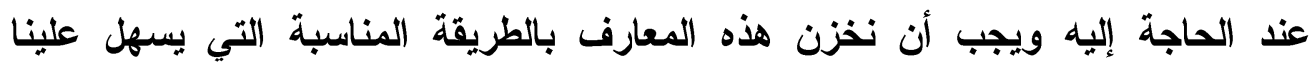
استرجاعها وفي حال خزنة بطريقة لا نستطيع استرجاعها نكون قد الد استخدمنا ذاكرتنا بطريقة خاطئة.

ويعرض اليسميث (Eliasmith, 2001) ) الذاكرة هي القدرة العامة أو الكلية لقدرتنا علي تفسير مدركات العالم وللمساعدة علي تنظيم الاستجابات للتغيرات التي تحدث

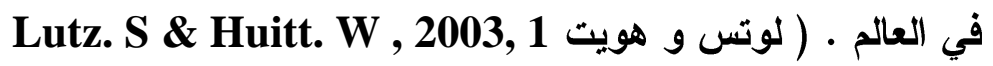

\footnotetext{
1 Memory

${ }^{2}$ Recognition

${ }^{3}$ Recall
} 
صعوبات التعلم النمائية ( الانتباهـ الادراك - الأكرة) وعلاقتها ببعض الاضطر ابات النفسية لدي عينة من تلاميذ المرحلة الابتائية بمحافظة قنا

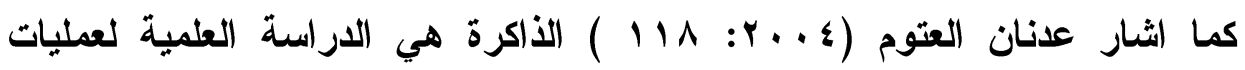

استقبال المعلومات وترميزها وتخزينها واستعادتها وقت أعان الحاجة.

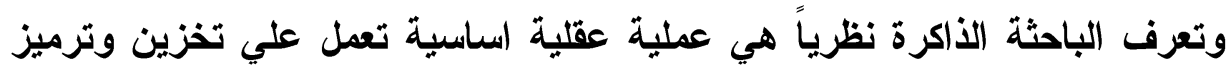

و استرجاع ما تم اكتسابه من المعلومات والخبرات السابقة. 
• احمد احد متولي.( • ( ؟). فاعلية برنامج تدريبي انتقائي تكاملي في خفض حدة أعر اض

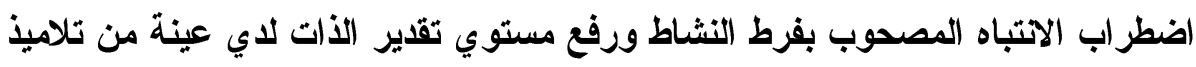

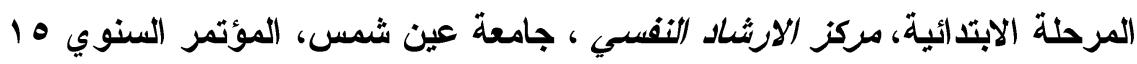

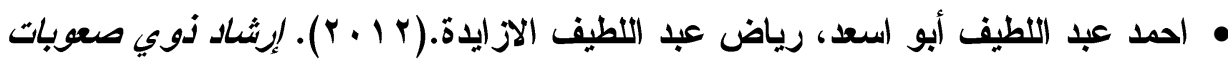

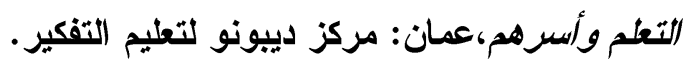

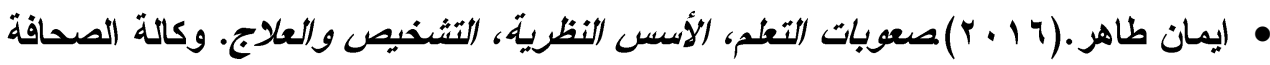
العربية ، القاهرة.

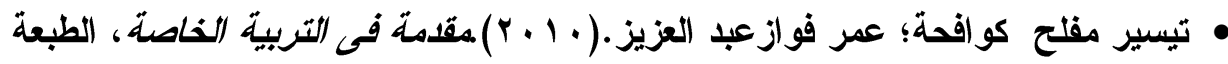

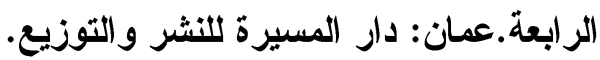

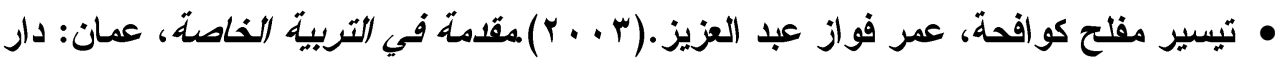

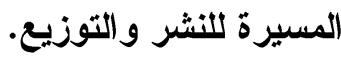

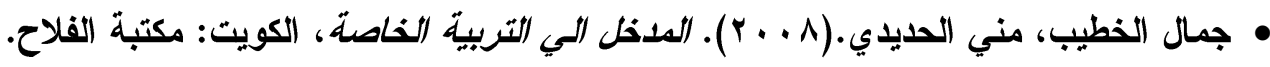

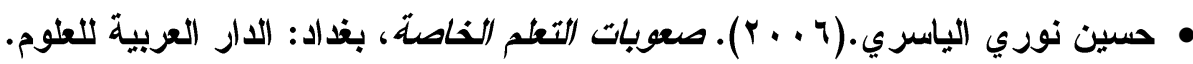

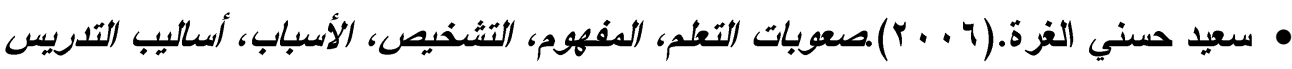
واستر|تيحيات العلاج، عمان: دار الثقافة.

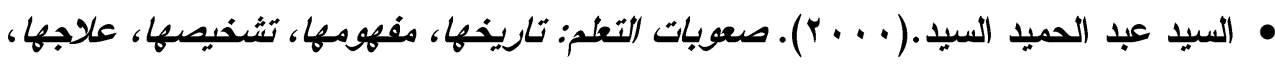
القاهرة: دار الفكر العربي.

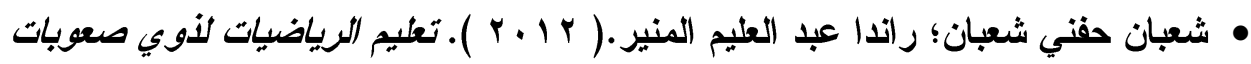

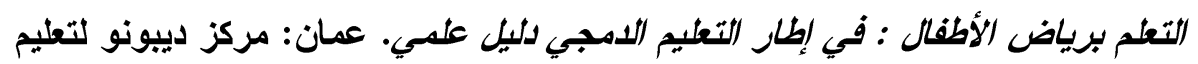
التفكير

• عانان يوسف العتوم.(ع . . r). علم الثفس المعرفي/ النظرية والتطبيق، عمان: دار المسيرة

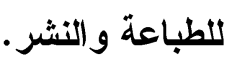

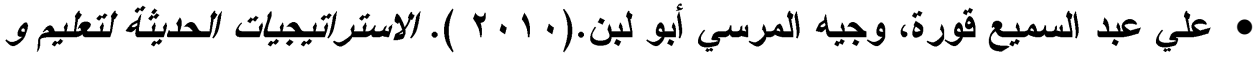

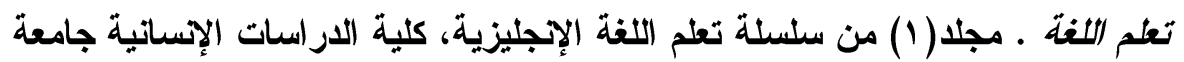
الازهر ، قسم المناهج وطرق التذريس.

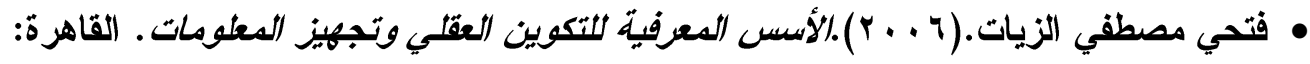
دار النشر للجامعات.

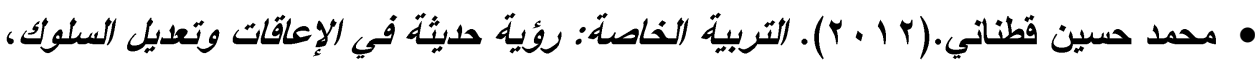
عمان: أمواج للطباعة و النشر . لمنيان. 
• مفيد حواثبين.(ع . . r). اثر التعزيز للمجموعات في تطوير الاتتباه لاي طلبة الصف الثاني

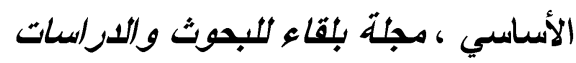

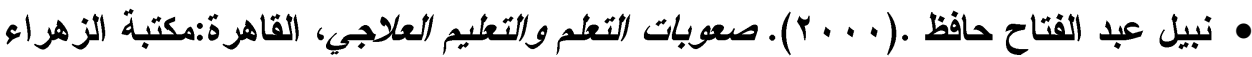
الشرق.

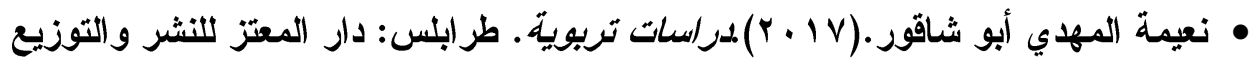

\section{المراجع الاجنبية:}

- Carla Vivas .(2008). ECKM2014-Proceedings of the 15th European conference on Knowledge Management: ECKM2014 . Academic Conferences and Publishing International. Italy.

- Eysenck \& Keane.(2005).cognitive psychology,Paris: Ithed, psychology, CTD, Nework.

- Fletcher, J. M., \& Foorman, B. R.(1994). Issues in Definition and measurement of learning Disabilities: The Needfor Early intervention .I.G.R Lyon (Ed). frames of refernce for the Assessment of learning Disbilitles, Baltimore: paul Brookes, 91(2), 263-292.

- Lerner, Janet W \& Beverley Johns .(2015).Learning Disabilities and Related Disabilities:Strategies for Successs, United State of America:Cengage Learning.

- Natalia M. Pylaeva \& Tatiana V. Akhutina .(2012).Overcoming Learning Disabilities. New York: Cambridge university Press .

- Raymond M. Stein ; Raymond M. Stein ; Melvin I. Freeman. (2012). The Ophthalmic Assistant: A Text for Allied and Associated Ophthalmic Personnel .China: Elsevier Health Science.

- Umilta C. (1998). Orienting of Attention: Handbook Neuropsychology, New York : Ma-Graw Hill

- Victorina O. Acero ; Evelyn S. Javier ; Herminia O. Castro .(2004) . Human Growth Development and Learning . London: Rex Book Store. Sampaloc, Manila.with ADHD, Journal of Attention Disorders, 19(1), 53-62. 